\title{
Benchmarking Density Functional Theory Approaches for the Description of Symmetry-Breaking in Long Polymethine Dyes
}

Rebecca L. Gieseking, Mahesh Kumar Ravva, Veaceslav Coropceanu, and Jean-Luc Bredas

J. Phys. Chem. C, Just Accepted Manuscript • DOI: 10.1021/acs.jpcc.6b02100 • Publication Date (Web): 25 Apr 2016

Downloaded from http://pubs.acs.org on May 3, 2016

\section{Just Accepted}

"Just Accepted" manuscripts have been peer-reviewed and accepted for publication. They are posted online prior to technical editing, formatting for publication and author proofing. The American Chemical Society provides "Just Accepted" as a free service to the research community to expedite the dissemination of scientific material as soon as possible after acceptance. "Just Accepted" manuscripts appear in full in PDF format accompanied by an HTML abstract. "Just Accepted" manuscripts have been fully peer reviewed, but should not be considered the official version of record. They are accessible to all readers and citable by the Digital Object Identifier (DOI®). "Just Accepted" is an optional service offered to authors. Therefore, the "Just Accepted" Web site may not include all articles that will be published in the journal. After a manuscript is technically edited and formatted, it will be removed from the "Just Accepted" Web site and published as an ASAP article. Note that technical editing may introduce minor changes to the manuscript text and/or graphics which could affect content, and all legal disclaimers and ethical guidelines that apply to the journal pertain. ACS cannot be held responsible for errors or consequences arising from the use of information contained in these "Just Accepted" manuscripts.

\section{ACS Publications}


Benchmarking Density Functional Theory Approaches for the Description of Symmetry-Breaking in Long Polymethine Dyes

\author{
Rebecca L. Gieseking, ${ }^{1}$ Mahesh Kumar Ravva, ${ }^{1,2}$ Veaceslav Coropceanu, ${ }^{1}$ \\ and Jean-Luc Brédas ${ }^{1,2, *}$
}

14

15

16

17

* Corresponding author: jean-luc.bredas@kaust.edu.sa; +966 (0) 128084855 


\begin{abstract}
Long polymethines are well-known experimentally to symmetry-break, which dramatically modifies their linear and nonlinear optical properties. Computational modeling could be very useful to provide insight into the symmetry-breaking process, which is not readily available experimentally; however, accurately predicting the crossover point from symmetric to symmetry-broken structures has proven challenging. Here, we benchmark the accuracy of several DFT approaches relative to $\operatorname{CCSD}(\mathrm{T})$ geometries. In particular, we compare analogous hybrid and long-range corrected (LRC) functionals to clearly show the influence of the functional exchange term. Although both hybrid and LRC functionals can be tuned to reproduce the CCSD(T) geometries, the LRC functionals are better performing at reproducing the geometry evolution with chain length and provide a finite upper limit for the gas-phase crossover point; these methods also provide good agreement with the experimental crossover points for more complex polymethines in polar solvents. Using an approach based on LRC functionals, a reduction in the crossover length is found with increasing medium dielectric constant, which is related to localization of the excess charge on the end groups. Symmetry-breaking is associated with the appearance of an imaginary frequency of $b_{2}$ symmetry involving a large change in the degree of bond-length alternation. Examination of the IR spectra show that short, isolated streptocyanines have a mode at $\sim 1200 \mathrm{~cm}^{-1}$ involving a large change in bond-length alternation; as the polymethine length or the medium dielectric increases, the frequency of this mode decreases before becoming imaginary at the crossover point.
\end{abstract}




\section{Introduction}

Linear $\pi$-conjugated molecules have been widely investigated over the past several decades for a variety of applications in nonlinear optics (NLO), including electro-optical modulation, ${ }^{1-3}$ twophoton absorption, ${ }^{4,5}$ and all-optical switching. ${ }^{6-9}$ In these molecules, the optical and NLO properties are highly dependent on the average degree of bond-length alternation (BLA; defined as the average difference between adjacent $\mathrm{C}-\mathrm{C}$ bond lengths) along the backbone. ${ }^{10-12}$ Of particular interest are polymethines, which consist of a bridge containing an odd number of methine $(\mathrm{CH})$ units between two identical end groups, see Figure 1.; these molecules are closedshell if they bear a single positive or negative charge. ${ }^{7,9}$ Short polymethines typically have (nearly) $\mathrm{C}_{2 \mathrm{v}}$ symmetry and BLA very close to zero, which is referred to as the cyanine limit. ${ }^{12-15}$ When this symmetry is maintained, polymethines have lower first excited-state energies ${ }^{12}$ and significantly sharper absorption peaks ${ }^{16,17}$ than polyenes (linear conjugated molecules with alternating double and single bonds) with equivalent conjugation lengths. These excited-state properties inherently give polymethines very large linear polarizabilities $\alpha$, negligibly small second-order polarizabilities $\beta$, and very large negative values of the real third-order polarizability $\operatorname{Re}(\gamma) .^{10,12}$

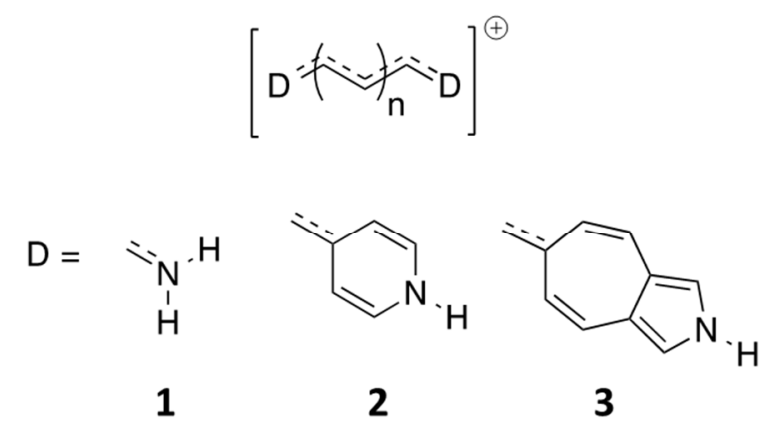

Figure 1. Chemical structures of the polymethines studied in this work. 
However, much experimental evidence ${ }^{18-27}$ underlines that when the dye is sufficiently long, polymethine geometries undergo a symmetry-breaking transition such that the excess charge becomes localized primarily on one end of the molecule. The absorption peaks then substantially broaden on the high-energy side or shift altogether to higher energy. ${ }^{18,22,28-30}$ This change is accompanied by a saturation or decrease in the magnitude of $\operatorname{Re}(\gamma)^{23}$ and the appearance of a non-zero $\beta .^{27}$ These modifications in the optical and NLO properties are consistent with an increase in BLA. Existence of such symmetry-broken structures with significant BLA has also been documented for several polymethines via experimental crystallographic data. ${ }^{19,31-37}$ The onset of symmetry-breaking can occur at polymethine bridge lengths as short as 3 carbons ${ }^{33}$ or as long as 13 carbons $^{22}$ depending on the polymethine end groups and environment. However, the broadened absorption peaks in solution are challenging to interpret. Experimental evidence to date has not been sufficient to clearly distinguish whether the broadened absorption peaks correspond to a single species or a mixture of symmetric and symmetry-broken polymethines; ${ }^{38}$ computational studies to date that examine the broadening of the absorption spectra have been limited to empirically selected approaches ${ }^{39}$ or parameterized models. ${ }^{40-42}$ In addition, it is unclear from current experiments how much change in the absorption spectra is expected for molecules near the crossover point, which corresponds to going from a single-well potential surface with one energetic minimum at a symmetric geometry and a BLA of zero to a doublewell potential where the symmetric structure is a transition structure between two symmetrybroken minima.

Computational studies of symmetry-breaking in polymethines can provide insight into polymethine symmetry-breaking, which is not readily accessible experimentally; however, 
accurately modeling the symmetry-breaking process has remained challenging. ${ }^{43,44}$ Polymethine symmetry-breaking behavior indeed depends on a delicate balance between, on the one hand, electronic couplings that favor delocalization of the wavefunction (delocalization of charge) and, on the other hand, Peierls-type electron-vibration interactions ${ }^{21,40,41}$ and interactions of the molecule with polar solvents ${ }^{41,44}$ that favor localization of the wavefunction (higher stability when localizing charge on an end group relative to the bridge). Since BLA is highly dependent on electron correlation, accurate prediction of BLA generally requires high-level methods such as $\operatorname{CCSD}(\mathrm{T}) .{ }^{45,46}$ However, determining the crossover point from a single-well to a double-well ground-state potential surface requires computing the optimized geometries and vibrational frequencies of large molecules, which limits the use of post-Hartree-Fock approaches due to the prohibitive computational costs. Although $\operatorname{CCSD}(\mathrm{T})$ geometries have previously been computed for streptocyanines (polymethines with $-\mathrm{NH}_{2}$ end groups; Figure 1., D $=1$ ), ${ }^{13}$ these calculations were limited to isolated ("gas-phase") polymethines with 13 or fewer carbons, in geometries with $\mathrm{C}_{2 \mathrm{v}}$ symmetry, and vibrational frequencies were not computed; thus, these calculations alone cannot provide any clear insight into the symmetry-breaking process.

Practical understanding of the dependence of symmetry-breaking on molecular structure is more readily accessible using lower-cost computational methods, such as Hartree-Fock (HF) and Density Functional Theory (DFT); these approaches provide upper and lower limits for the crossover point. HF, known to over-localize the wavefunction and thus yield BLA values that are too large $e^{47-49}$ and underestimate the symmetry-breaking point, ${ }^{50}$ gives a streptocyanine crossover point at a bridge length of 19 carbons in the gas phase. ${ }^{43}$ On the other hand, typical DFT approaches over-delocalize the wavefunction and predict too small BLA values ${ }^{47-49}$ and maintain 
symmetric structures to very large bridge lengths; ${ }^{51,52}$ for example, the commonly-used functional B3LYP retains symmetric streptocyanine structures to at least 39 carbons. ${ }^{43,44}$ The crossover point can be tuned by varying the percentage of exact HF exchange in a hybrid functional; ${ }^{39,48}$ however, empirical selection of an appropriate DFT functional is challenging because of the lack of validation relative to a high-level computational standard $^{13}$ and the aforementioned complexities in comparing to experimental results. This broad range between the lower limit (19 carbons) and upper limit (infinite) for the crossover point limits our understanding of the symmetry-breaking process. Long-range corrected (LRC) functionals, which divide the exchange term into a short-range term that is primarily DFT exchange and a long-range HF exchange term, are able to better reproduce the BLA values in polyenes than do conventional hybrid functionals; ${ }^{53}$ however, to the best of our knowledge, the accuracy of such functionals in the polymethine geometries and symmetry-breaking has not yet been assessed.

Here, we benchmark the accuracy of analogous hybrid and LRC functionals relative to CCSD(T) geometries $^{13}$ and show that LRC functionals are better able to reproduce the evolution of BLA with polymethine length. Importantly, tuned LRC functionals provide a finite upper limit for the gas-phase streptocyanine crossover point. We use such a functional to examine the effects of the dielectric medium and the chemical structure of the end group on the crossover point. We also examine in detail the vibrational modes associated with symmetry breaking.

\section{Computational Methodology}

Geometry optimizations and frequency calculations were performed using several DFT functionals combining PBE with HF exchange in conjunction with the $6-31 \mathrm{G}^{*}$ basis set. The first 
functional was the conventional hybrid PBE $\alpha,{ }^{54,55}$ known for $\alpha=0.25$ as PBE0. In this functional, the exchange term is a linear combination of PBE and HF contributions:

$$
E_{\mathrm{xc}}^{\mathrm{PBE} \alpha}=\alpha E_{\mathrm{X}}^{\mathrm{HF}}+(1-\alpha) E_{\mathrm{x}}^{\mathrm{PBE}}+E_{\mathrm{c}}^{\mathrm{PBE}}
$$

In contrast, in the long-range corrected functional $\omega \mathrm{PBEh}^{56}{ }^{56}$ the exchange term is primarily DFT exchange for short-range electron-electron interactions and HF exchange at long range, divided according to the error function:

$$
\frac{1}{r}=\frac{\operatorname{erfc}(\omega r)}{r}+\frac{\operatorname{erf}(\omega r)}{r}
$$

The form of the functional is then

$$
E_{\mathrm{xc}}^{\omega \mathrm{PBEh}}=0.2 E_{\mathrm{x}}^{\mathrm{SR}-\mathrm{HF}}+0.8 E_{\mathrm{x}}^{\mathrm{SR}-\mathrm{PBE}}+E_{\mathrm{x}}^{\mathrm{LR}-\mathrm{HF}}+E_{\mathrm{c}}^{\mathrm{PBE}}
$$

where $\omega$ is the tunable range-separation parameter.

The $\% \mathrm{HF}(\alpha)$ for the hybrid PBE $\alpha$ functional and the range-separation parameter $(\omega)$ for the LRC $\omega$ PBEh functional were tuned to reproduce BLA obtained at the $\operatorname{CCSD}(\mathrm{T}) / 6-31 \mathrm{G}^{*}$ level of theory considered here as a benchmark. Additional calculations based on the PBE0 functional and on the $\omega \mathrm{PBEh}$ functional with the default $\omega=0.2 \mathrm{bohr}^{-1}$ value were also performed. The optimized geometries were computed for $\mathrm{C}_{2 \mathrm{v}}$-symmetric structures $\left(\mathrm{C}_{2}\right.$-symmetric structures for streptocyanines of 33 carbons and longer due to loss of planarity at the nitrogen atoms) and, in 
the symmetry-broken polymethines, for symmetry-broken structures with $\mathrm{C}_{\mathrm{s}}$ symmetry. Calculations were performed both for isolated molecules and using the SM8 model of implicit solvation. $^{57}$ Atomic charges were computed via the natural bond order (NBO) population analysis for all optimized geometries at the $\omega \mathrm{PBEh} / 6-31 \mathrm{G}^{*}$ level. These calculations were performed using Q-Chem 4.1. ${ }^{58}$

\section{Results and Discussion}

\subsection{Geometric structures and tuning of functionals}

As described in the Introduction, the nature of the functional, particularly in terms of the treatment of HF exchange, dramatically affects BLA and the localization/delocalization of charge in $\pi$-conjugated systems. Thus, we first evaluate the accuracy of various functionals relative to the high-level $\operatorname{CCSD}(\mathrm{T}) / 6-31 \mathrm{G}^{*}$ standard. ${ }^{13}$ In particular, we examine the PBE $\alpha$ and WPBEh functionals, as detailed in Section 2. Although the tuning of LRC functionals is typically done based on ionization potential and/or electron affinity, this procedure has previously been shown to poorly reproduce the evolution of BLA. ${ }^{53}$ In contrast, selection of hybrid functionals to date for polymethines has primarily been based on empirical comparison to experimental absorption spectra. ${ }^{39,48}$ Here, we instead tune $\alpha$ and $\omega$ to reproduce the $\operatorname{CCSD}(\mathrm{T}) / 6-31 \mathrm{G}^{*}$ BLA values of model streptocyanines. As BLA reflects the degree of delocalization of the wavefunction, a functional that provides accurate BLA values is expected to also accurately predict the symmetry-breaking behavior. 
The most commonly used definition of BLA in linear conjugated systems is the average BLA, defined as the difference between the average lengths of single and double bonds along the molecular backbone:

$$
\text { average BLA }=\frac{\left(b_{1-2}+b_{3-4}+\cdots\right)-\left(b_{2-3}+b_{4-5}+\cdots\right)}{(N-1) / 2}
$$

where $b_{x-y}$ is the bond length between atoms $\mathrm{C}_{\mathrm{x}}$ and $\mathrm{C}_{\mathrm{y}}$ and $N$ is the number of carbon atoms in the polymethine backbone. Polyenes with alternating single and double bonds have average BLAs of around $0.1 \AA$, whereas $\mathrm{C}_{2 \mathrm{v}}$-symmetric polymethines have average BLAs of exactly zero by symmetry. Since the average BLA cannot distinguish between different geometries of the symmetric polymethines, we focus instead on the absolute average $B L A$, defined as the average of the absolute differences between adjacent carbon-carbon bond lengths along the polymethine backbone:

$$
\text { absolute average BLA }=\frac{\left|b_{1-2}-b_{2-3}\right|+\left|b_{2-3}-b_{3-4}\right|+\cdots}{(N-2)}
$$

The absolute average BLA is equal to zero only when all carbon-carbon bond lengths are identical in length, e.g., in the $\mathrm{C}_{2 \mathrm{v}}$-symmetric geometry of the 3-carbon streptocyanine (which contains only two carbon-carbon bonds that are identical by symmetry), but is non-zero in longer polymethines. In some cases, it is also useful to examine the terminal $B L A$, defined as the difference between the two carbon-carbon bond lengths nearest one end group (for symmetric polymethines, the terminal BLA values near each end group are identical): 


$$
\text { terminal } \mathrm{BLA}=\left|b_{1-2}-b_{2-3}\right|
$$

The tuned $\alpha$ and $\omega$ values were obtained by first performing geometry optimizations for several values of the tuning parameters, then iteratively selecting new trial values using linear interpolation/extrapolation from the nearest two points, see Figure 2. This procedure was repeated until the absolute average BLA was within $10^{-5} \AA$ of the $\operatorname{CCSD}(\mathrm{T}) / 6-31 \mathrm{G}^{*}$ values. Within the ranges of the tuning parameters considered, the evolution of BLA with the tuning parameter is nearly linear, so only a handful of iterations were required to complete the tuning. From this procedure, tuned values of $\alpha=0.456$ and $\omega=0.160 \mathrm{bohr}^{-1}$ were obtained. We note the large tuned $\alpha$ value is comparable to that of functionals like BH\&HLYP that have previously shown polymethine crossover points in reasonable agreement with experimental results; ${ }^{43}$ these results are also consistent with previous observations that functionals with large contributions of HF exchange predict excited-state energies in short streptocyanines more accurately. ${ }^{59}$ 

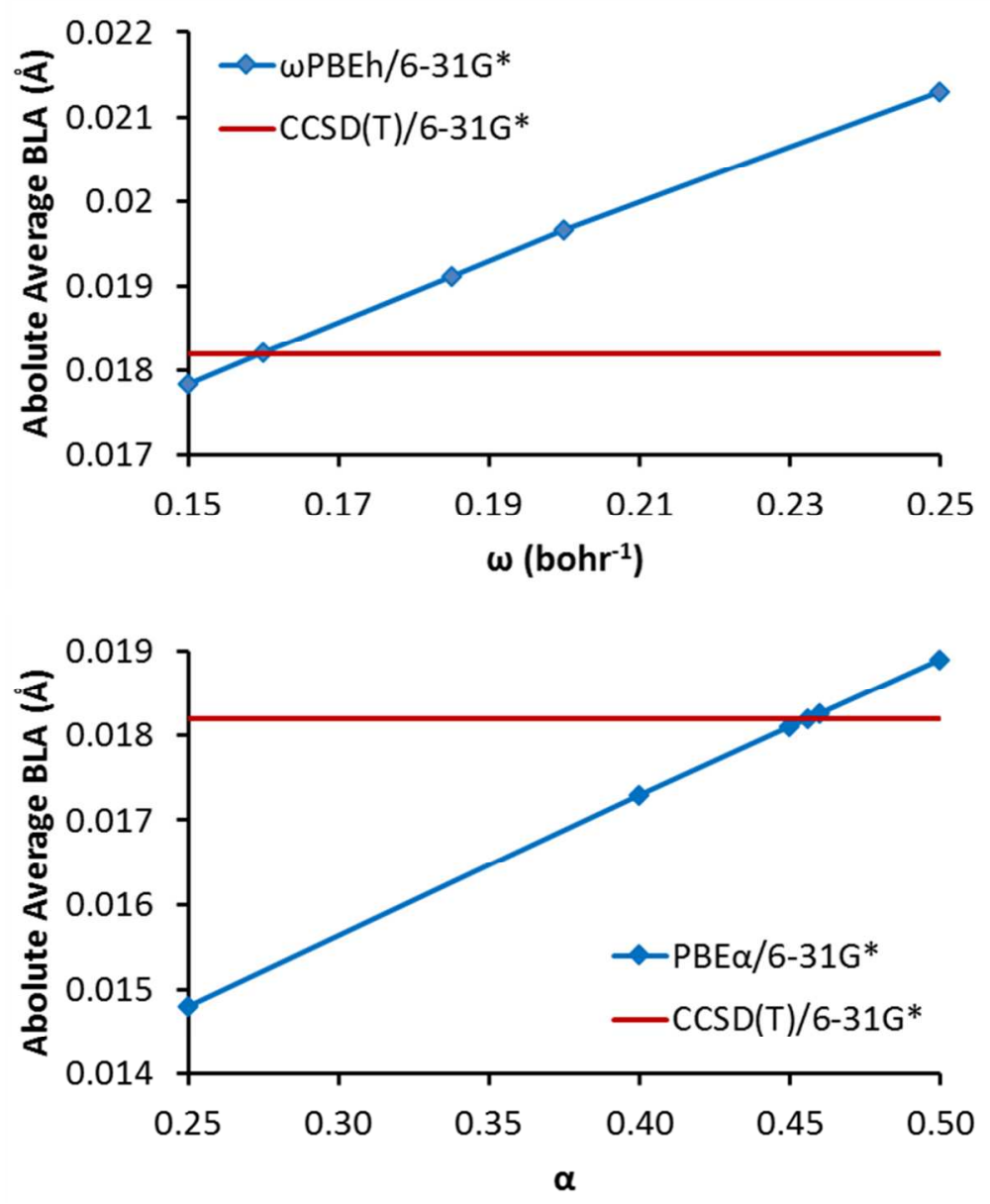

Figure 2. Absolute average BLA of 13-carbon streptocyanines as a function of (top) $\omega$ and (bottom) $\alpha$.

To examine the effects of functional type and tuning on BLA, we compare four functionals: (1) the hybrid functional PBE $\alpha$ with the default $\alpha$ of 0.25 (25\% HF exchange; PBE0); (2) PBE $\alpha$ with a value of $\alpha(=0.456)$ tuned to reproduce the absolute average BLA of the 13-carbon streptocyanine at the $\operatorname{CCSD}(\mathrm{T}) / 6-31 \mathrm{G}^{*}$ level; (3) the $\mathrm{LRC} \omega \mathrm{PBEh}$ functional with the default $\omega$ of $0.200 \mathrm{bohr}^{-1}$; and (4) the $\omega$ PBEh functional with a value of $\omega\left(=0.160 \mathrm{bohr}^{-1}\right)$ tuned to reproduce the absolute average BLA of the 13-carbon streptocyanine at the $\operatorname{CCSD}(\mathrm{T}) / 6-31 \mathrm{G}^{*}$ level. All of these functionals involve a combination of PBE and HF exchange, which allows for direct comparison of LRC vs. hybrid functionals. 
The comparison of the absolute average BLAs is shown in Figure 3.. The PBE0 calculations (purple crosses) substantially underestimate BLA at all molecular lengths, which is consistent with over-delocalization of the wavefunction; this is a typical result for hybrid functionals with a small amount of HF exchange. In contrast, the default LRC functional $\omega$ PBEh (red squares) slightly overestimates BLA; this is in line with the known ability of such functionals to reproduce well the polyene BLA value. ${ }^{53}$
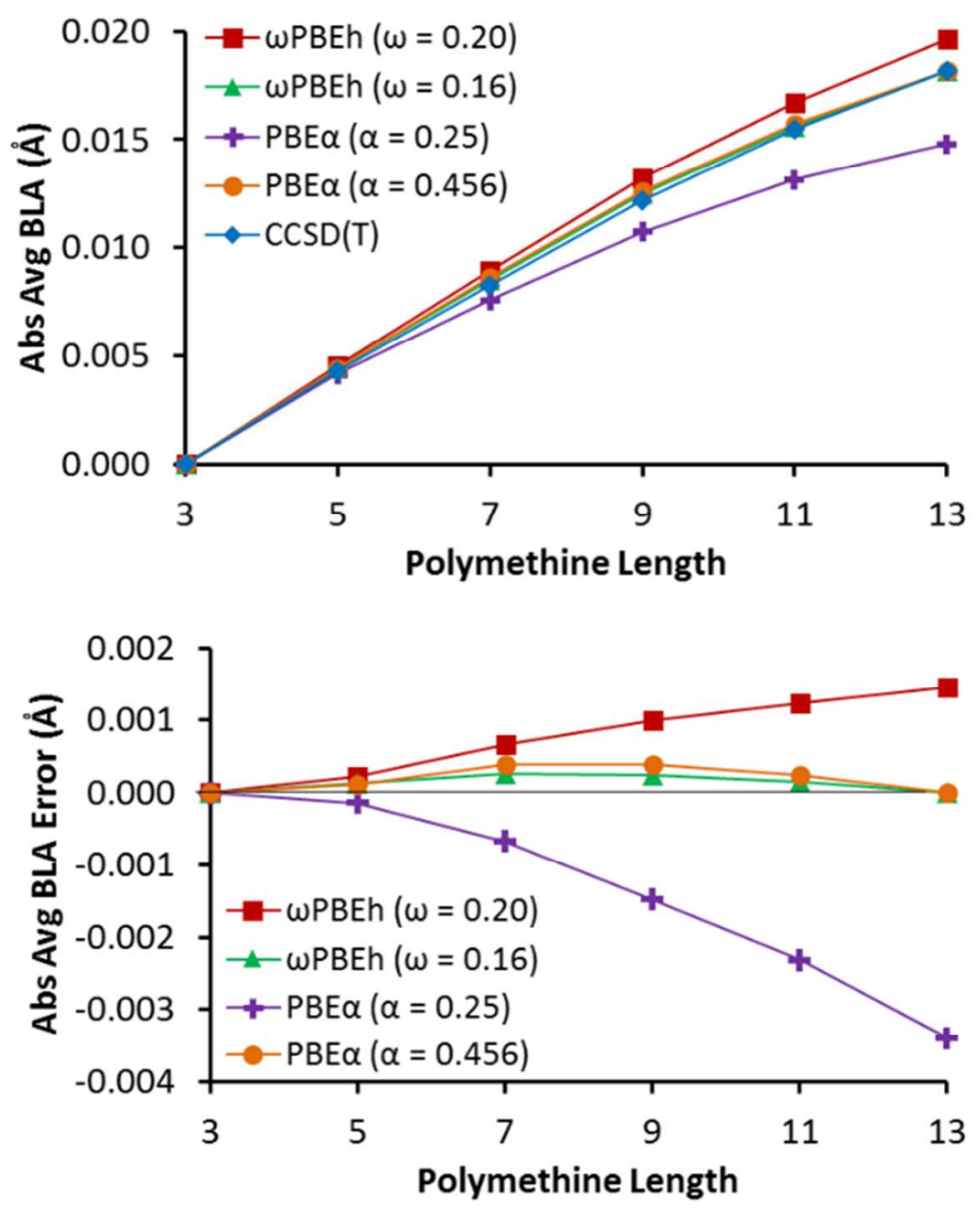

Figure 3. Absolute average BLA (top) and deviation from CCSD(T)/6-31G* BLA (bottom) for the default and tuned functionals. 
Since the tuned hybrid (orange circles) and LRC (green triangles) functionals have values of $\alpha$ and $\omega$ selected to reproduce the $\operatorname{CCSD}(\mathrm{T}) / 6-31 \mathrm{G}^{*} \mathrm{BLA}$ of the $13 \mathrm{C}$ cyanine, the very small error at that length merely shows the efficacy of the tuning procedure. The key question is whether a single value of $\alpha$ or $\omega$ can accurately reproduce the evolution of BLA over an appreciable range of molecular lengths. For the shorter polymethines (5-11 carbons), both tuned functionals slightly overestimate BLA. We note that the deviations in absolute average BLA for both functionals are well within crystallographic accuracy $(0.001 \AA)$; however, the tuned LRC functional yields a more accurate evolution of BLA than does the hybrid functional. Although, to our knowledge, the $\operatorname{CSSD}(\mathrm{T}) \mathrm{BLA}$ values have not been computed for longer polymethines, the trends in the deviations suggest that both tuned functionals will underestimate BLA for polymethines longer than 13 carbons and that the tuned hybrid will give a more significant underestimation than will the tuned LRC functional.

When the polymethine length is extended beyond 13 carbons, BLA continues to increase, see Figure 4. Between roughly 13 and 21 carbons, all functionals show a decrease in the slope of BLA; this change in slope is particularly pronounced for the default PBE $\alpha$. This is related to a slower increase in the terminal BLA for longer polymethines; regardless of molecular length, the terminal BLA never increases beyond the polyene limit of $\sim 0.1 \AA$. Among the four functionals, the default LRC consistently provides the largest BLA, whereas the default PBE0 hybrid consistently has the smallest BLA. As expected from the shorter polymethines, a crossover in the BLA of the two tuned functionals is observed: the tuned LRC gives smaller BLA than the tuned hybrid for polymethines shorter than 13 carbons, whereas the reverse is true for longer polymethines. In combination with the earlier comparison to $\operatorname{CCSD}(\mathrm{T}) \mathrm{BLA}$ for shorter 
polymethines, these trends suggest that the tuned LRC functional gives a lower limit for the $\operatorname{CCSD}(\mathrm{T}) \mathrm{BLA}$ in polymethines longer than 13 carbons.

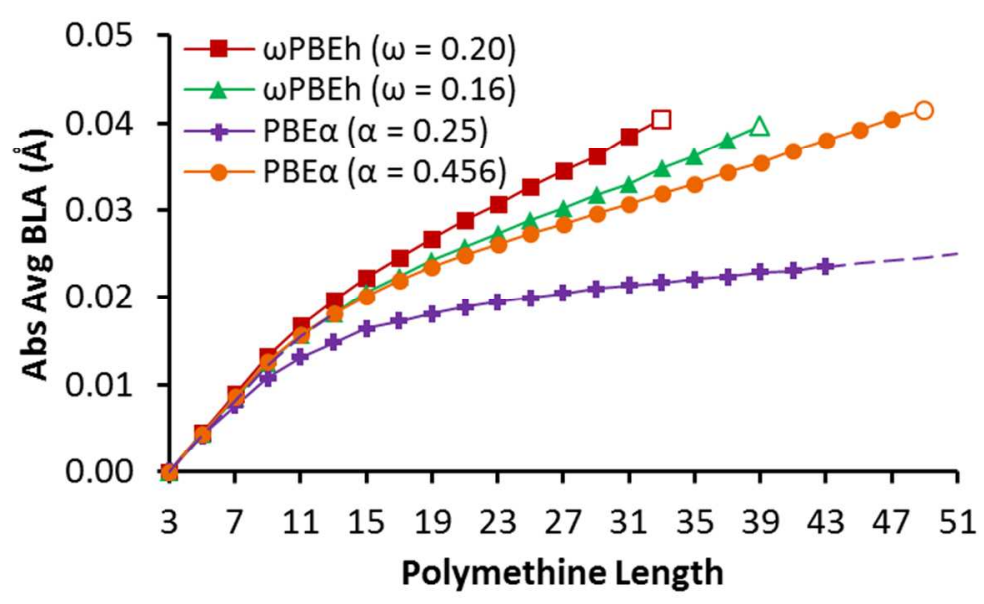

Figure 4. Absolute average BLA of streptocyanines. The open symbols indicate the shortest symmetry-broken polymethines at each level of theory.

The choice of functional also affects the bridge length for which symmetry-breaking occurs. For all functionals except PBE0, structures were computed by increasing the bridge length until a $b_{2}$ symmetry vibration mode (in the $\mathrm{C}_{2 \mathrm{v}}$-symmetric geometry) with imaginary frequency is observed, i.e., until the first symmetry-broken geometry is reached. In Figure 4., the open symbols correspond to the shortest symmetry-broken system at each level of theory. The results show that the symmetric to symmetry-broken crossover point correlates with BLA: the functionals that yield larger BLA for moderately long polymethines show symmetry breaking at shorter bridge lengths. In fact, all three functionals have crossover points at BLA within a narrow range between 0.039 and $0.042 \AA$. Taking the tuned LRC functional as a lower limit for BLA, this suggests that the tuned LRC functional provides an upper limit for the CCSD(T) gas-phase 
crossover point. Significantly, because of the comparison to a high-level standard, these calculations provide a finite upper limit of 39 carbons for the gas-phase crossover point. These calculations point to the fact that even in the gas phase, the stabilization of charge due to the $\mathrm{NH}_{2}$ end groups is sufficient to cause symmetry-breaking at finite bridge lengths. As expected, the crossover point in the gas phase occurs at much longer bridge lengths than is observed experimentally in polar solvents; ${ }^{18,22,28}$ the effect of solvation on cyanine length at the crossover point is examined in the following section. As will be discussed later, this functional also provides reasonable agreement with experimental evidence of symmetry breaking in polar solvents for polymethines with more complex end groups; this agreement confirms that this functional can provide useful information to understand experimental data.

\subsection{Effect of Solvation}

Since we have validated the accuracy of the methodology based on the tuned $\omega$ PBEh functional, the remainder of this paper will focus on using this functional (with $\omega$ of $0.160 \mathrm{bohr}^{-1}$ ) to understand the effects of environment and chemical structure on the crossover point and on related properties such as the charge distribution and vibrational modes. Experimental data demonstrate that the length at which symmetry-breaking occurs depends on the solvent polarity. ${ }^{18,19}$ Here, we use the SM8 implicit solvation model to study the effect of the environment on the streptocyanine charge distributions and geometries.

The effect of dielectric media corresponding to hexane $(\varepsilon=1.88)$ and DMSO $(\varepsilon=46.8)$ on the polymethine geometries is shown in Figure 5. As expected, the crossover point decreases dramatically with increasing medium polarity, from 39 carbons in the gas phase to 27 carbons in 
hexane and 15 carbons in DMSO. Here, in contrast to the earlier comparison of different functionals, the crossover points occur at very different BLA values in the different dielectric environments.

\begin{abstract}
At each bridge length, the polymethines in more polar environments have much smaller BLA. In the gas phase, the terminal $\mathrm{N}-\mathrm{C}$ bond is shorter than any of the $\mathrm{C}-\mathrm{C}$ bonds, but its length is somewhat closer to that of a typical N-C single bond between $\operatorname{sp}^{2}$ atoms $(\sim 1.37 \AA)$ than to that of a typical $\mathrm{N}=\mathrm{C}$ double bond $(\sim 1.27 \AA)$. The terminal $\mathrm{C}$ - $\mathrm{C}$ bonds are the shortest $\mathrm{C}-\mathrm{C}$ bonds along the molecular backbone, indicating that these bonds have the most double-bond character; the alternation becomes muted when approaching the center of the molecule as the adjacent bond orders become more equal. An increasing medium dielectric constant leads to a decrease in the $\mathrm{N}-\mathrm{C}$ bond length, suggesting that the $\mathrm{N}-\mathrm{C}$ bonds acquire more double-bond character; the C-C bond lengths correspondingly become more equal, see Figure 5.
\end{abstract}



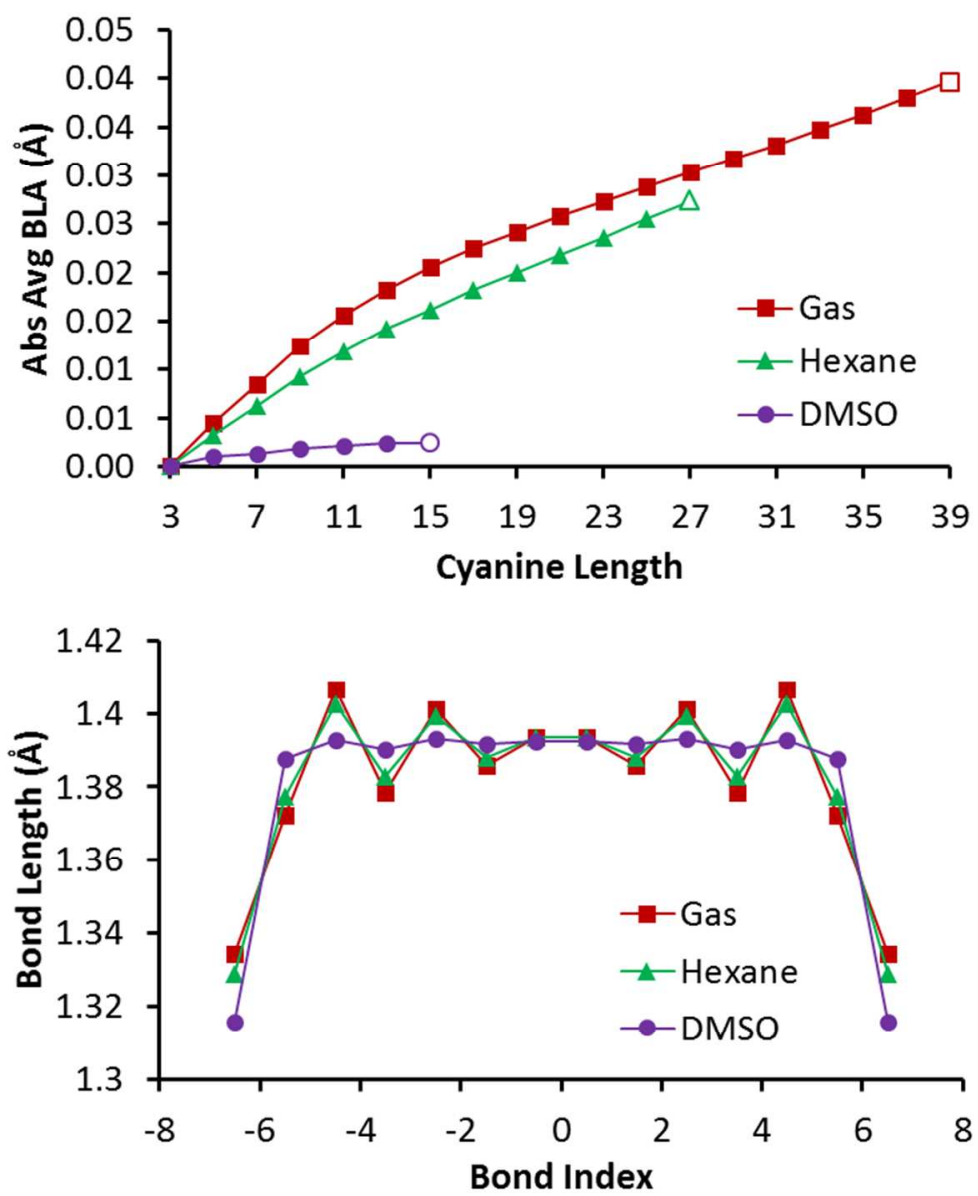

Figure 5. (Top) Absolute average BLA of polymethines at the tuned $\omega$ PBEh $(\omega=0.16)$ level as a function of medium dielectric. (Bottom) Bond lengths of the 13-carbon polymethine; the terminal lengths in the plot are the terminal $\mathrm{N}-\mathrm{C}$ bond lengths.

The decrease in BLA with increasing dielectric constant can be understood in terms of the resonance forms that contribute to the electronic structure. As shown in Figure 6., the changes in bond lengths reflect changes in which resonance forms have the most significant contributions to the electronic structure. When the structures with charges on the nitrogen atoms dominate, the N$\mathrm{C}$ bonds have significant double-bond character and are relatively short, and all of the $\mathrm{C}$ - $\mathrm{C}$ bonds have very similar bond orders and lengths, as is seen in DMSO. When a significant amount of charge is instead present on the bridge, the $\mathrm{N}-\mathrm{C}$ bonds have more single-bond character and the terminal C-C bonds have more double-bond character, as is seen in the gas phase. 
As a reflection of these resonance forms, it has long been known that polymethines inherently have significant charge alternation along the molecular backbone. ${ }^{13,14,60,61}$ We can further confirm that the variations in bond length are due to changes in the resonance-form contributions by examining the charge alternation along the molecular backbone (where we sum the hydrogen charges into the corresponding heavy atoms). The atomic charges follow the expected pattern of alternation, with positive charges on the end groups and odd-numbered carbons and slight negative charges on the even-numbered carbons, as illustrated in Figure 7.. The majority of the charge is localized on the end groups and the terminal carbon atoms; on the central atoms, there is significant charge alternation but relatively little net charge. Because of inductive effects, the charge on the terminal carbons is more positive than that on the end groups, and the charge on the nitrogen does not clearly reflect the contribution of the resonance form with the charge on the end group; as a result, it is more appropriate to focus primarily on the summed charge on each terminal $-\mathrm{CHNH}_{2}$ group. As the molecular length increases (while maintaining $\mathrm{C}_{2 \mathrm{v}}$ symmetry), there are more atoms over which to delocalize the charge, and the charges on the end groups and 
terminal carbon atoms become smaller. This is consistent with a smaller contribution of resonance forms with charge on the end groups and the increase in BLA described earlier.
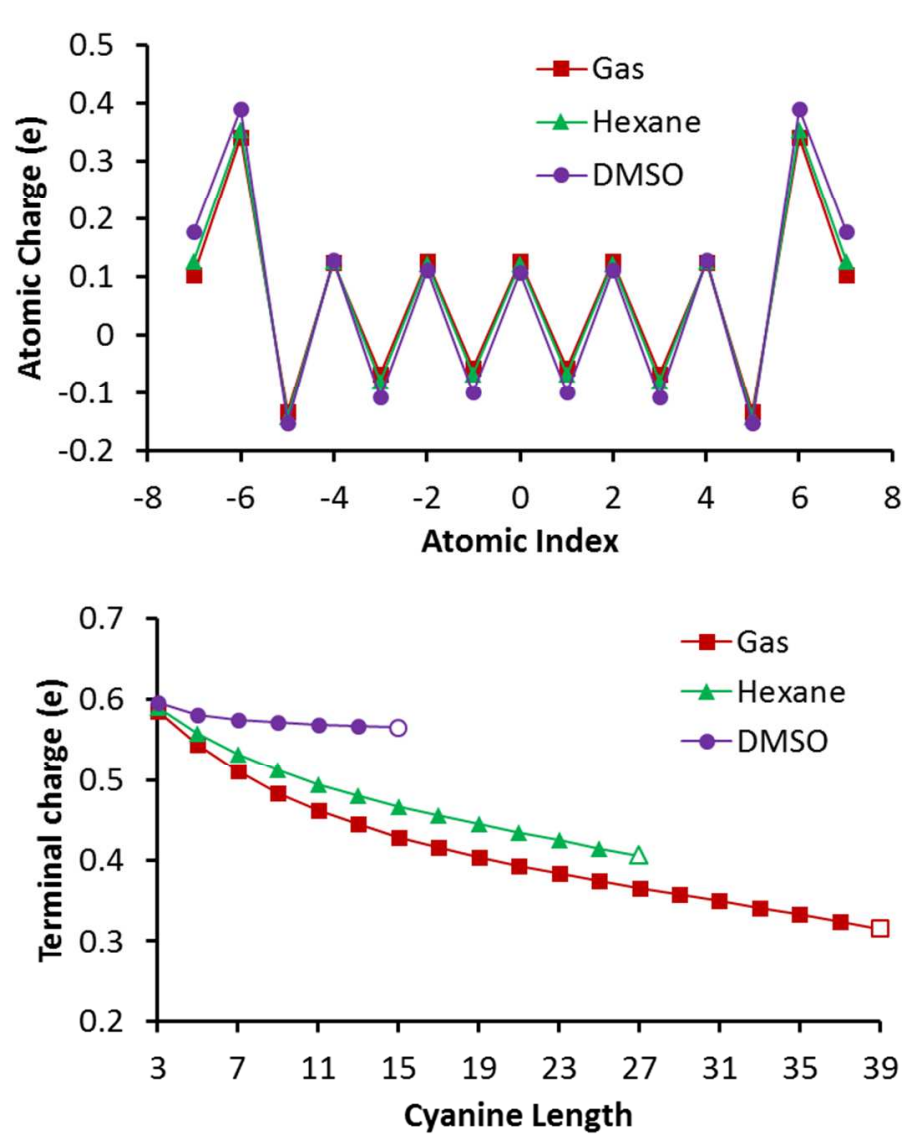

Figure 7. (Top) Atomic charges of the 13-carbon streptocyanine with the hydrogen charges summed into the heavy atoms. (Bottom) Charge on the terminal $-\mathrm{CHNH}_{2}$ groups.

Consideration of implicit solvation increases the positive charge on the terminal groups by up to 0.08 electrons. This is unsurprising, since solvent typically stabilizes the localization of charge. The impact of solvent on the molecular charges increases with increasing molecular length; in simulations in DMSO, the charge on the end groups is nearly independent of molecular length. 
This is consistent with maintaining short N-C bonds and BLA very close to zero in DMSO until the crossover point.

The differences in the relationship between BLA and symmetry-breaking when changing the solvent vs. the functional (as described in the previous Section) is related to the factors leading to symmetry-breaking. As described in the Introduction, symmetry-breaking occurs when the charge-localized structure is more stable than the charge-delocalized structure. When the functional is changed, polymethines of the same length have the same external environment and thus a similar energetic cost of localizing charge; however, the electronic couplings between adjacent carbons along the backbone are different. The electronic structure of the polymethines corresponds to a positively-charged soliton centered at the middle of the polymethine bridge, with significant charge also localized on the end groups. ${ }^{21,29,44,62}$ When the electronic couplings between adjacent carbons are smaller, corresponding to a narrower soliton with more charge localized near the center of the molecule and thus larger BLA; this leads to symmetry-breaking at shorter bridge lengths. In contrast, when the dielectric constant is increased, the primary effect is to stabilize the localization of charge on the end groups. As seen from the resonance forms in Figure 6., more localization of charge on the end groups reduces BLA; this also leads to a shorter crossover point.

To this point, we have focused solely on polymethine geometric structures with $\mathrm{C}_{2 \mathrm{v}}$ symmetry, identifying symmetry-broken structures by the presence of $a b_{2}$ symmetry vibrational mode with an imaginary frequency. ${ }^{63}$ To gain more insight into the origins of this mode, we focus here on the evolution of the vibrational properties when approaching the crossover point, then turn to the 
geometries and properties of the symmetry-broken structures. As discussed previously, symmetry-breaking is associated with an increase in BLA and localization of charge on one molecular end; this symmetry-broken geometry pattern can only be achieved via a distortion of the $\mathrm{C}_{2 \mathrm{v}}$-symmetric structure along a vibrational coordinate of $\mathrm{b}_{2}$ symmetry. Since stretching of $\mathrm{C}$ $\mathrm{C}$ bonds is required to change BLA, we focus on the vibrational modes with frequencies $<2000$ $\mathrm{cm}^{-1}$.

The symmetry-breaking process involves a large change in the molecular dipole moment; as a result the IR intensities (directly proportional to the square of the derivative of the dipole moment with respect to displacement) of the modes most strongly associated with symmetrybreaking are expected to be large. The streptocyanines, particularly the longer molecules, have many carbon-carbon stretching modes; however, only a few modes have large IR intensities. In the short polymethines, two modes have particularly large IR intensities: one around $1250 \mathrm{~cm}^{-1}$ and one around $1600 \mathrm{~cm}^{-1}$ (Figure 8.; images of the corresponding modes as a function of polymethine length are provided in the SI). These modes both involve large changes in BLA (as presented in the SI); the lower-frequency mode involves synchronous motion of each methine unit, whereas the higher-frequency mode involves bending of each methine unit. Across the full range of molecular lengths considered, the two modes with largest IR intensities involve large changes in BLA regardless of the exact frequencies of the modes. 
(a)

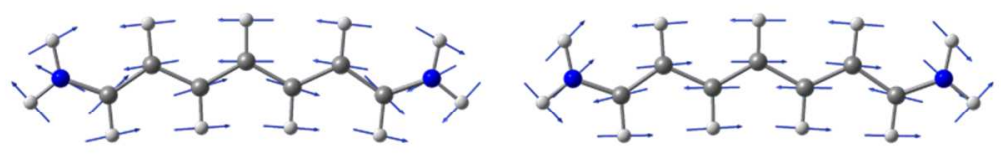

(b)

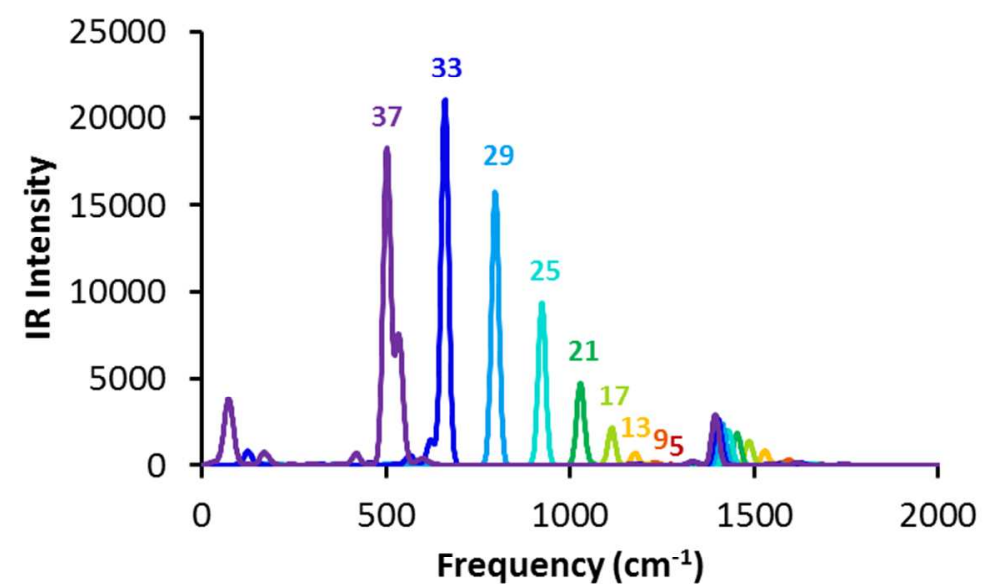

(c)

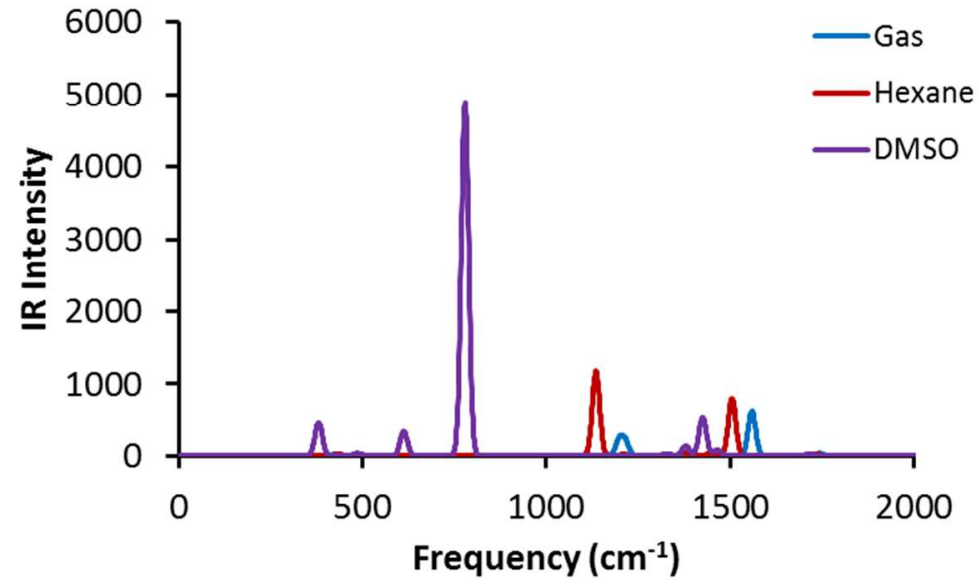

Figure 8. (a) Vibrational modes for the 7-carbon streptocyanine at the $\omega \mathrm{PBEh} / 6-31 \mathrm{G} *$ level $\left(\omega=0.16\right.$ ) with frequencies of (left) $1252 \mathrm{~cm}^{-1}$ and (right) $1622 \mathrm{~cm}^{-1}$. Infrared spectra of (b) gas-phase streptocyanines as a function of length and (c) the 11-carbon streptocyanine in solvent.

At short molecular lengths, the frequencies of these high-IR-intensity modes in the gas phase are relatively constant with length; from 5 to 13 carbons, the frequencies of the two modes each decrease by less than $100 \mathrm{~cm}^{-1}$. However, as the molecular length continues to increase, the frequencies (particularly of the lower-frequency mode) decrease more dramatically; the 
frequency decreases to $<1000 \mathrm{~cm}^{-1}$ at 23 carbons and to $\sim 500 \mathrm{~cm}^{-1}$ at 37 carbons before becoming imaginary at 39 carbons. For polymethines of 33 carbons and longer we note the appearance of multiple low-frequency modes that show a significant decrease in energy with a concomitant increase in the IR intensity upon increase of the molecular length; in these systems, we focus on the highest-intensity of the mode as labeled in Figure 8.. Based on the frequencies of these models, in short polymethines the potential energy surface for changing BLA has a force constant typical of $\mathrm{C}-\mathrm{C}$ bond stretching. As molecular length increases, the potential energy surface gradually becomes flatter before transitioning to a double-well potential at 39 carbons.

When considering changes in the dielectric environment at a constant molecular length, very similar effects are seen. Most molecules still have two modes with large IR intensities, with some mixing of low-frequency modes near the crossover point as described previously. There is relatively little change in the frequency and IR intensity for the mode at $1400-1600 \mathrm{~cm}^{-1}$; much larger changes are seen for the lower-frequency mode. As described previously, consideration of implicit solvation dramatically affects the length at which the molecular symmetry breaks. The crossover points occur at 15 carbons in both DMSO and water and 27 carbons in hexane (Table 1.). As the molecular length increases beyond the crossover point, the imaginary frequency of the mode associated with symmetry-breaking becomes larger. Interestingly, the projection of the normal modes computed in solvent on the gas-phase modes indicates a significant similarity (see Figure S12) between the imaginary modes and those exhibiting the largest IR activity in the gasphase. For instance, the imaginary modes of 15-carbon and 17-carbon polymethines derived correspond to gas-phase modes at 1149 and $1113 \mathrm{~cm}^{-1}$, respectively. 
Table 1. Streptocyanine imaginary frequencies, energetic difference $\Delta E$ between the $C_{2 v}$ and $C_{s}$ structures, and BLA at the $\omega$ PBEh/6-31G* level.

\begin{tabular}{|c|c|c|c|c|c|c|}
\hline \multirow[t]{2}{*}{$N$} & \multirow[t]{2}{*}{ Solvent } & \multirow{2}{*}{$\begin{array}{l}\text { Imaginary } \\
\text { Frequency } \\
\quad\left(\mathrm{cm}^{-1}\right)\end{array}$} & \multirow{2}{*}{$\begin{array}{c}\Delta \mathrm{E} \\
(\mathrm{kcal} / \mathrm{mol})\end{array}$} & \multicolumn{3}{|c|}{ BLA $(\AA)$} \\
\hline & & & & $\begin{array}{c}\text { C }_{2 v} \text { Abs. } \\
\text { Avg. }\end{array}$ & $\begin{array}{c}\text { C }_{\text {s }} \text { Abs. } \\
\text { Avg. }\end{array}$ & $C_{\text {s Avg. }}$ \\
\hline 39 & - & 89.6 & -0.020 & 0.040 & 0.041 & 0.016 \\
\hline 41 & - & 175.2 & -0.086 & 0.041 & 0.044 & 0.024 \\
\hline 27 & Hexane & 293.2 & -0.068 & 0.027 & 0.036 & 0.028 \\
\hline 29 & Hexane & 456.8 & -0.340 & 0.029 & 0.044 & 0.040 \\
\hline 15 & DMSO & 645.5 & -0.380 & 0.002 & 0.049 & 0.044 \\
\hline 17 & DMSO & 974.6 & -1.095 & 0.002 & 0.054 & 0.056 \\
\hline
\end{tabular}

For the streptocyanines with imaginary frequencies, we also compare the $\mathrm{C}_{2 \mathrm{v}}$-symmetric transition states to the $\mathrm{C}_{\mathrm{s}}$-symmetric energetic minima. For the gas-phase streptocyanines, the energetic stabilization upon symmetry-breaking is extremely small, more than two orders of magnitude smaller than thermal energy at room temperature $(0.6 \mathrm{kcal} / \mathrm{mol})$. The $\mathrm{C}_{2 \mathrm{v}}$-symmetric structures have fairly large absolute average BLAs owing to symmetrically-distributed alternation far from the molecular center. Upon symmetry-breaking, the change in the molecular geometry is small, as evidenced by the small average BLA and small increase in the absolute average BLA. In solvent, the energetic stabilization upon symmetry-breaking is more significant and is larger for molecules further beyond the crossover point; in fact, for the 17-carbon streptocyanine in DMSO, the stabilization upon symmetry-breaking is greater than thermal energy, suggesting that symmetric structures are unlikely to be observed at moderate temperatures. The changes in geometry upon symmetry-breaking are also more significant, transitioning from structures where all carbon-carbon bond lengths are nearly equal in length to 
structures with substantial alternation across the entire molecule. This is consistent with the large shift and broadening in the experimental absorption spectra upon symmetry-breaking.

\subsection{Effect of End Groups}

To compare the computed bridge length corresponding to the symmetry-related crossover point to that observed experimentally, we now turn to polymethines with larger conjugated end groups that act as stronger donors (Figure 1.). As mentioned previously, it is challenging to directly compare the computed crossover point to the experimental absorption spectra; however, it is possible to determine whether the computed crossover points are at a bridge length qualitatively similar to those at which broadening of the experimental absorption spectrum is observed.

The extended conjugation in the larger end groups decreases the length of the crossover point. In polar solvents, the computed crossover points are comparable to the lengths at the onset of broadening of the experimental absorption spectra. The pyridinium polymethines in DMSO experimentally show substantial broadening of the absorption peak at 9 carbons and a shift of the absorption peak to higher energy at 13 carbons, ${ }^{22}$ which agrees well with our computed results (Table 2.). The azaazulene polymethines show noticeable broadening of their absorption peaks at 9 carbons in dichloromethane (DCM) and 7 carbons in acetonitrile (ACN) ${ }^{28}$ slightly longer than the computed lengths of symmetry-breaking. This discrepancy may be due to simplifications to the end group used in the calculations (several alkyl groups, which may shield the solvent from the end groups, were not considered). However, the overall consistency with experiment demonstrates that our approach is reliable enough to provide insight into the symmetry-breaking process in polymethines. 
Table 2. Bridge lengths $(N)$ of polymethine crossover points at the $\omega \mathrm{PBEh} / 6-31 G^{*}$ level.

\begin{tabular}{|c|c|c|c|c|c|}
\hline D & $\begin{array}{c}\text { Vacuum } \\
(\boldsymbol{\varepsilon}=\mathbf{1})\end{array}$ & $\begin{array}{c}\text { Hexane } \\
(\boldsymbol{\varepsilon}=\mathbf{1 . 8 8})\end{array}$ & $\begin{array}{c}\text { DCM } \\
(\boldsymbol{\varepsilon}=\mathbf{8 . 9 3})\end{array}$ & $\begin{array}{c}\text { ACN } \\
(\boldsymbol{\varepsilon}=\mathbf{3 5 . 6 8})\end{array}$ & $\begin{array}{c}\text { DMSO } \\
(\boldsymbol{\varepsilon}=\mathbf{4 6 . 8 2})\end{array}$ \\
\hline Amino (1) & 39 & 27 & - & - & 15 \\
\hline Pyridinium (2) & 29 & 19 & - & - & 11 \\
\hline Azaazulene (3) & 29 & 19 & 9 & 7 & 7 \\
\hline
\end{tabular}

We note that in DMSO, the crossover point decreases with increasing end group size when considering the number of carbons in the bridge as shown in Table 2.. However, when counting the total number of adjacent carbons between the terminal nitrogen atoms, the crossover point is at a nearly constant length: 15, 17, and 17 carbons, respectively, for the amino, pyridinium, and azaazulene end groups. This suggests that when the donor atoms are moderately chemically similar (all $\mathrm{sp}^{2}$ nitrogen atoms), the physical length is important to determining the crossover point. Further work is needed to understand how other chemical changes to the end groups can affect the crossover point.

\section{Conclusions}

A computational understanding of the symmetry-breaking process in polymethines is needed to understand how molecular structure and environment affect the crossover point from symmetric to symmetry-broken structures. Here, we have shown that the streptocyanine structures at the tuned $\omega \mathrm{PBEh} / 6-31 \mathrm{G}^{*}$ level are in good agreement with the $\operatorname{CCSD}(\mathrm{T}) / 6-31 \mathrm{G}^{*}$ structures. ${ }^{13}$ Comparison of the tuned $\omega \mathrm{PBEh} / 6-31 \mathrm{G}^{*}$ results to the experimental absorption spectra for 
polymethines with conjugated end groups provides further evidence that this computational approach can be used to provide insight into symmetry-breaking.

\begin{abstract}
Our calculations also provide insight into the molecular structures of the polymethines and the changes upon symmetry-breaking. In the gas phase, polymethines retain $C_{2 v}$ symmetry to very long bridge lengths but acquire significant alternation in the carbon-carbon bond lengths far from the molecular center; little change in this geometric pattern is observed upon symmetry-breaking. In contrast, in polar solvents, short polymethines have more charge localized in their end groups and very small BLA while symmetry is retained. However, symmetry-breaking occurs at much shorter bridge lengths, and a much larger change in BLA occurs upon symmetry-breaking because of the stabilization of charge on one of the end groups. The transition from symmetric to symmetry-broken geometry is associated with a gradual shift in the $\mathrm{C}-\mathrm{C}$ stretching mode associated with a large change in BLA toward an imaginary frequency. The imaginary modes calculated in solution show substantial similarities with the gas-phase modes that have the largest IR intensities.
\end{abstract}

\begin{abstract}
By validating this method at a high level of theory, we have provided a clear finite upper limit for the length of gas-phase streptocyanine symmetry-breaking. This method can be further applied to understand the chemical effects of how structural changes determine the length of the crossover point.
\end{abstract}




\section{Supporting Information}

Details of the vibrational modes of streptocyanines in the gas phase and in DMSO, including frequencies, intensities, and images of the atomic displacements. This information is available free of charge via the Internet at http://pubs.acs.org.

\section{Acknowledgments \\ This work was primarily supported by the National Institute of Neurological Disorders and Stroke of the National Institutes of Health under Award Number R21NS084353. We acknowledge stimulating discussions with Drs. T. Körzdörfer, S.R. Marder, and J.W. Perry.}

\section{References}

(1) Kim, T.-D.; Kang, J.-W.; Luo, J.; Jang, S.-H.; Ka, J.-W.; Tucker, N.; Benedict, J. B.; Dalton, L.; Gray, T.; Overney, R. M. et al. Ultralarge and Thermally Stable Electro-Optic Activities from Supramolecular Self-Assembled Molecular Glasses. J. Am. Chem. Soc. 2007, 129, 488-489.

(2) Dalton, L. R.; Benight, S. J.; Johnson, L. E.; Knorr, D. B.; Kosilkin, I.; Eichinger, B. E.; Robinson, B. H.; Jen, A. K. Y.; Overney, R. M. Systematic Nanoengineering of Soft Matter Organic Electro-Optic Materials. Chem. Mater. 2011, 23, 430-445.

(3) Kwon, O. P.; Kwon, S.-J.; Jazbinsek, M.; Seo, J.-Y.; Kim, J.-T.; Seo, J.-I.; Lee, Y. S.; Yun, H.; Günter, P. Phenolic Polyene Crystals with Tailored Physical Properties and Very Large Nonlinear Optical Response. Chem. Mater. 2011, 23, 239-246.

(4) He, G. S.; Tan, L.-S.; Zheng, Q.; Prasad, P. N. Multiphoton Absorbing Materials: Molecular Designs, Characterizations, and Applications. Chem. Rev. 2008, 108, 1245-1330.

(5) Pawlicki, M.; Collins, H. A.; Denning, R. G.; Anderson, H. L. Two-Photon Absorption and the Design of Two-Photon Dyes. Angew. Chem. Int. Ed. 2009, 48, 3244-3266.

(6) Hales, J. M.; Matichak, J.; Barlow, S.; Ohira, S.; Yesudas, K.; Bredas, J. L.; Perry, J. W.; Marder, S. R. Design of Polymethine Dyes with Large Third-Order Optical Nonlinearities and Loss Figures of Merit. Science 2010, 327, 1485-1488.

(7) Hales, J. M.; Barlow, S.; Kim, H.; Mukhopadhyay, S.; Brédas, J.-L.; Perry, J. W.; Marder, S. R. Design of Organic Chromophores for All-Optical Signal Processing Applications. Chem. Mater. 2014, 26, 549-560.

(8) Barlow, S.; Brédas, J. L.; Getmanenko, Y. A.; Gieseking, R. L.; Hales, J. M.; Kim, H.; Marder, S. R.; Norwood, R. A.; Perry, J. W.; Risko, C. et al. Polymethine Materials with Solid- 
State Third-Order Optical Susceptibilities Suitable for All-Optical Signal-Processing Applications. Mater. Horiz. 2014, 1, 577-581.

(9) Gieseking, R. L.; Mukhopadhyay, S.; Risko, C.; Marder, S. R.; Brédas, J.-L. 25th Anniversary Article: Design of Polymethine Dyes for All-Optical Switching Applications: Guidance from Theoretical and Computational Studies. Adv. Mater. 2014, 26, 68-84.

(10) Marder, S. R.; Gorman, C. B.; Meyers, F.; Perry, J. W.; Bourhill, G.; Brédas, J. L.; Pierce, B. M. A Unified Description of Linear and Nonlinear Polarization in Organic Polymethine Dyes. Science 1994, 265, 632-635.

(11) Marder, S. R.; Perry, J. W.; Bourhill, G.; Gorman, C. B.; Tiemann, B. G.; Mansour, K. Relation between Bond-Length Alternation and Second Electronic Hyperpolarizability of Conjugated Organic Molecules. Science 1993, 261, 186-189.

(12) Meyers, F.; Marder, S. R.; Pierce, B. M.; Bredas, J. L. Electric Field Modulated Nonlinear Optical Properties of Donor-Acceptor Polyenes: Sum-over-States Investigation of the Relationship between Molecular Polarizabilities (Alpha, Beta, and Gamma) and Bond Length Alternation. J. Am. Chem. Soc. 1994, 116, 10703-10714.

(13) Jacquemin, D. New Cyanine Dyes or Not? Theoretical Insights for Model Chains. $J$. Phys. Chem. A 2011, 115, 2442-2445.

(14) Kachkovski, A. D.; Dekhtyar, M. L. Electronic Properties of Polymethine Systems. Part 4: Electronic Structure of Polymethine Chain. Dyes Pigm. 1996, 30, $43-54$.

(15) Groth, P. On the Disordered Crystal Structure of Bis(Dimethylamino)-Heptamethinium Cloride Tetrahydrate at Room Temperature. Acta Chem. Scand., Ser. B 1987, 41, 547-550.

(16) Kachkovsky, A.; Pilipchuk, N.; Kurdyukov, V.; Tolmachev, A. Electronic Properties of Polymethine Systems. 10. Electron Structure and Absorption Spectra of Cyanine Bases. Dyes Pigm. 2006, 70, 212-219.

(17) Fu, J.; Padilha, L. A.; Hagan, D. J.; Van Stryland, E. W.; Przhonska, O. V.; Bondar, M. V.; Slominsky, Y. L.; Kachkovskii, A. Molecular Structure - Two-Photon Absorption Property Relations in Polymethine Dyes. J. Opt. Soc. Am. B 2007, 24, 56-66.

(18) Lepkowicz, R. S.; Przhonska, O. V.; Hales, J. M.; Fu, J.; Hagan, D. J.; Van Stryland, E. W.; Bondar, M. V.; Slominsky, Y. L.; Kachkovski, A. D. Nature of the Electronic Transitions in Thiacarbocyanines with a Long Polymethine Chain. Chem. Phys. 2004, 305, 259-270.

(19) Bouit, P.-A.; Aronica, C.; Toupet, L.; Guennic, B. L.; Andraud, C.; Maury, O. Continuous Symmetry Breaking Induced by Ion Pairing Effect in Heptamethine Cyanine Dyes: Beyond the Cyanine Limit. J. Am. Chem. Soc. 2010, 132, 4328-4335.

(20) Yu, A.; Tolbert, C. A.; Farrow, D. A.; Jonas, D. M. Solvatochromism and Solvation Dynamics of Structurally Related Cyanine Dyes. J. Phys. Chem. A 2002, 106, 9407-9419.

(21) Tolbert, L. M.; Ogle, M. E. How Far Can a Carbanion Delocalize? 13c Nmr Studies of Soliton Model Compounds. J. Am. Chem. Soc. 1990, 112, 9519-9527.

(22) Tolbert, L. M.; Zhao, X. Beyond the Cyanine Limit: Peierls Distortion and Symmetry Collapse in a Polymethine Dye. J. Am. Chem. Soc. 1997, 119, 3253-3258.

(23) Wernke, W.; Pfeiffer, M.; Johr, T.; Lau, A.; Grahn, W.; Johannes, H.-H.; Dahne, L. Increase and Saturation of the Third Order Hyperpolarizabilities in Homologous Series of Symmetric Cyanines. Chem. Phys. 1997, 216, 337-347.

(24) Fuyura, K.; Inagaki, Y.; Torii, H.; Furukawa, Y. T., Mitsuo. Vibrational Analysis of a Strongly Correlated System, Pentamethine Streptocyanine Dye, Based on Observed Infrared and Raman Spectra and Density Functional Calculations. J. Phys. Chem. A 1998, 102, 8413-8421. 
(25) Fuyura, K.; Torii, H.; Furukawa, Y.; Tasumi, M. Vibrational Spectra and Structures of Long-Chain Streptocyanine Dyes: Effects of Electron-Vibration Interactions and Vibrational Polarizabilities. J. Phys. Chem. A 2000, 104, 11203-11211.

(26) Marks, A. F.; Noah, A. K.; Sahyun, M. R. V. Bond-Length Alternation in Symmetrical Cyanine Dyes. J. Photochem. Photobiol., A 2001, 139, 143-149.

(27) Zoueu, J. T.; Fiorini-Debuisschert, C.; Charra, F.; Nunzi, J.-M. Transient SecondHarmonic Generation in a Cyanine Liquid Dye Solution. Chem. Phys. Lett. 2006, 419, 454-457. (28) Przhonska, O. V.; Hu, H.; Webster, S.; Bricks, J. L.; Viniychuk, A. A.; Kachkovski, A. D.; Slominsky, Y. L. Electronic Transitions in a Series of 2-Azaazulene Polymethine Dyes with Different П-Conjugation Lengths. Chem. Phys. 2013, 411, 17-25.

(29) Kachkovski, O. D.; Tolmachov, O. I.; Slominskii, Y. L.; Kudinova, M. O.; Derevyanko, N. O.; Zhukova, O. O. Electronic Properties of Polymethine Systems 7: Soliton Symmetry Breaking and Spectral Features of Dyes with a Long Polymethine Chain. Dyes Pigm. 2005, 64, 207-216.

(30) Vasyluk, S. V.; Viniychuk, O. O.; Poronik, Y. M.; Kovtun, Y. P.; Shandura, M. P.; Yashchuk, V. M.; Kachkovsky, O. D. Breaking of Symmetrical Charge Distribution in Xanthylocyanine Chromophores Detecting by Their Absorption Spectra. J. Mol. Struct. 2011, 990, 6-13.

(31) Dahne, L.; Reck, G. Deformation of Polymethine Structures by Intermolecular Interactions. Angew. Chem. Int. Ed. 1995, 34, 690-692.

(32) Honda, M.; Katayama, C.; Tanaka, J. Charge Density Distribution of a Polymethine Cyanine Dye: (5-Dimethylamino-2,4-Pentadienylidene)Dimethylammonium Perchlorate. Acta Crystallogr. 1986, B42, 90.

(33) Barlow, S.; Henling, L. M.; Day, M. W.; Marder, S. R. Effect of the End-Groups Upon Delocalisation in Polymethines: The First Crystallographically Characterised Bond-Alternated Cyanine. Chem. Commun. 1999, 1567-1568.

(34) Tanaka, J.; Tanaka, M.; Hayakawa, M. Electronic Spectra of Single Crystals of 1,1'Diethyl-2,2'-Cyanine Iodide, Bromide, and Chloride. Bull. Chem. Soc. Jpn. 1980, 53, 3109-3119. (35) Wheatley, P. J. 659. The Crystallography of Some Cyanine Dyes. J. Chem. Soc. 1959, 3245 .

(36) Wheatley, P. J. 821. The Crystallography of Some Cyanine Dyes. Part Ii. The Molecular and Crystal Structure of the Ethanol Solvate of 3,3?-Diethylthiacarbocyanine Bromide. J. Chem. Soc. 1959, 4096.

(37) Yoshioka, H.; Nakatsu, K. Crystal Structures of Two Photographic Sensitizing Dyes, 1,1'-Diethyl-2,2'-Cyanine Bromide and 1,1'-Diethyl-4,4'-Cyanine Bromide Chem. Phys. Lett. 1971, 11, 255-258.

(38) Hu, H.; Przhonska, O. V.; Terenziani, F.; Painelli, A.; Fishman, D.; Ensley, T. R.; Reichert, M.; Webster, S.; Bricks, J. L.; Kachkovski, A. D. et al. Two-Photon Absorption Spectra of a near-Infrared 2-Azaazulene Polymethine Dye: Solvation and Ground-State Symmetry Breaking. Phys. Chem. Chem. Phys. 2013, 15, 7666-7678.

(39) Masunov, A. E.; Anderson, D.; Freidzon, A. Y.; Bagaturyants, A. A. Symmetry-Breaking in Cationic Polymethine Dyes: Part 2. Shape of Electronic Absorption Bands Explained by the Thermal Fluctuations of the Solvent Reaction Field. J. Phys. Chem. A 2015, 119, 6807-6815. (40) Sissa, C.; Jahani, P. M.; Soos, Z. G.; Painelli, A. Essential State Model for Two-Photon Absorption Spectra of Polymethine Dyes. ChemPhysChem 2012, 13, 2795-2800. 
(41) Terenziani, F.; Przhonska, O. V.; Webster, S.; Padilha, L. A.; Slominskii, Y. L.; Davydenko, I. G.; Gerasov, A. O.; Kovtun, Y. P.; Shandura, M. P.; Kachkovski, A. D. et al. Essential-State Model for Polymethine Dyes: Symmetry Breaking and Optical Spectra. J. Phys. Chem. Lett. 2010, 1, 1800-1804.

(42) Petrenko, A.; Stein, M. Molecular Reorganization Energy as a Key Determinant of JBand Formation in J-Aggregates of Polymethine Dyes. The journal of physical chemistry. A 2015, 119, 6773-6780.

(43) Fabian, J. Symmetry-Lowering Distortion of near-Infrared Polymethine Dyes — a Study by First-Principles Methods. J. Mol. Struct.: THEOCHEM 2006, 766, 49-60.

(44) Iordanov, T. D.; Davis, J. L.; Masunov, A. E.; Levenson, A.; Przhonska, O. V.; Kachkovski, A. D. Symmetry Breaking in Cationic Polymethine Dyes, Part 1: Ground State Potential Energy Surfaces and Solvent Effects on Electronic Spectra of Streptocyanines. Int. J. Quantum Chem. 2009, 109, 3592-3601.

(45) Jacquemin, D.; Perpète, E. A.; Scalmani, G.; Frisch, M. J.; Kobayashi, H.; Adamo, C. Assessment of the Efficiency of Long-Range Corrected Functionals for Some Properties of Large Compounds. J. Chem. Phys. 2007, 126, 144105.

(46) Jacquemin, D.; Adamo, C. Bond Length Alternation of Conjugated Oligomers: Wave Function and Dft Benchmarks. J. Chem. Theory Comput. 2010, 7, 369-376.

(47) Brédas, J. L. Relationship between Band Gap and Bond Length Alternation in Organic Conjugated Polymers. J. Chem. Phys. 1985, 82, 3808-3811.

(48) Yesudas, K. Cationic Cyanine Dyes: Impact of Symmetry-Breaking on Optical Absorption and Third-Order Polarizabilities. Phys. Chem. Chem. Phys. 2013, 15, 19465-19477.

(49) Ho Choi, C.; Kertesz, M.; Karpfen, A. The Effects of Electron Correlation on the Degree of Bond Alternation and Electronic Structure of Oligomers of Polyacetylene. The Journal of Chemical Physics 1997, 107, 6712.

(50) Gieseking, R. L.; Mukhopadhyay, S.; Risko, C.; Marder, S. R.; Bredas, J. L. Effect of Bulky Substituents on Thiopyrylium Polymethine Aggregation in the Solid State: A Theoretical Evaluation of the Implications for All-Optical Switching Applications. Chem. Mater. 2014, 26, 6439-6447.

(51) Gieseking, R. L.; Mukhopadhyay, S.; Shiring, S. B.; Risko, C.; Bredas, J. L. Impact of Bulk Aggregation on the Electronic Structure of Streptocyanines: Implications for the SolidState Nonlinear Optical Properties and All-Optical Switching Applications. J. Phys. Chem. C 2014, 118, 23575-23585.

(52) Coropceanu, V.; Lambert, C.; Nöll, G.; Brédas, J. L. Charge-Transfer Transitions in Triarylamine Mixed-Valence Systems: The Effect of Temperature. Chemical Physics Letters 2003, 373, 153-160.

(53) Korzdorfer, T.; Parrish, R. M.; Sears, J. S.; Sherrill, C. D.; Bredas, J. L. On the Relationship between Bond-Length Alternation and Many-Electron Self-Interaction Error. $J$. Chem. Phys. 2012, 137, 124305.

(54) Adamo, C.; Barone, V. Toward Reliable Density Functional Methods without Adjustable Parameters: The Pbe0 Model. J. Chem. Phys. 1999, 110, 6158.

(55) Perdew, J. P.; Ernzerhof, M.; Burke, K. Rationale for Mixing Exact Exchange with Density Functional Approximations. J. Chem. Phys. 1996, 105, 9982.

(56) Shuai, Z.; Peng, Q. Excited States Structure and Processes: Understanding Organic LightEmitting Diodes at the Molecular Level. Phys. Rep. 2014, 537, 123-156. 
(57) Soos, Z. G.; Galvao, D. S.; Etemad, S. Fluorescence and Excited-State Structure of Conjugated Polymers. Adv. Mater. 1994, 6, 280-287.

(58) Shao, Y.; Gan, Z.; Epifanovsky, E.; Gilbert, A. T. B.; Wormit, M.; Kussmann, J.; Lange, A. W.; Behn, A.; Deng, J.; Feng, X. et al. Advances in Molecular Quantum Chemistry Contained in the Q-Chem 4 Program Package. Mol. Phys. 2014, 113, 184-215.

(59) Jacquemin, D.; Zhao, Y.; Valero, R.; Adamo, C.; Ciofini, I.; Truhlar, D. G. Verdict: Time-Dependent Density Functional Theory "Not Guilty" of Large Errors for Cyanines. $J$. Chem. Theory Comput. 2012, 8, 1255-1259.

(60) Dahne, S.; Kulpe, S. Abh. Akad. Wiss. DDR, Abt. Math. Naturwiss., Tech. 1977, 8, 1-128.

(61) Dahne, S.; Ranft, J. Z. Z. Phys. Chem. 1963, 224, 65.

(62) Craw, J. S.; Reimers, J. R.; Bacskay, G. B.; Wong, A. T.; Hush, N. S. Solitons in Finiteand Infinite-Length Negative-Defect Trans-Polyacetylene and the Corresponding Brooker (Polymethinecyanine) Cations. I. Geometry. Chem. Phys. 1992, 167, 77-99.

(63) At the wPBEh/6-31G* level, some long polymethines (15-carbon or longer in the gas phase; 19-carbon or longer in hexane) have small imaginary frequencies $(>100 \mathrm{~cm}-1)$ of b1 symmetry corresponding to bending of the polymethine away from planarity. As there is no energetic stabilization upon displacement along these modes, these modes are assumed to be due to numerical error and have been neglected. 


\section{TOC image}

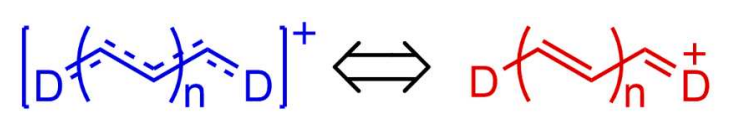

Hybrid vs. Long-Range Corrected Functional 